\title{
Better reasoning about software engineering activities
}

\author{
Tim Menzies ${ }^{1}$ \\ University of British Comlumbia \\ timemenzies.com
}

\author{
James D. Kiper ${ }^{2}$ \\ Miami University \\ kiperjd@muohio.edu
}

\begin{abstract}
Software management oracles often contain numerous subjective features. At each subjective point, a range of behaviors is possible. Stochastic simulation samples a subset of the possible behaviors. After many such stochastic simulations, the TAR2 treatment learner can find control actions that have (usually) the same impact despite the subjectivity of the oracle.
\end{abstract}

\section{Introduction}

Experience with software management can be encoded into an management oracle that can offer advise on how to structure a software project. Three components are required for such an oracle. Firstly, a knowledge source is required. Secondly, a syntax is required to encode that knowledge source. Thirdly, an interpreter is required to execute the syntax. In the case of subjective software engineering knowledge, this interpreter must be able to handle degrees of belief.

This paper reports experience with one such oracle. The knowledge source was a detailed description of CMM2 [3, p125-191]. We used CMM2 since, in our experience, many organizations can achieve at least this level. CMM2 is less concerned with issues of (e.g.) which design pattern to apply, than with what overall project structure should be implemented. Improving CMM2-style decisions is important since in early software lifecycle, many CMM2-style decisions effect the resource allocation for the rest of the project.

The JANE language was used to encode the knowledge source. In order to handle degrees of belief, JANE assigns a Chances weight to all of its propositions. This weight is a subjective judgement and so, for each run, the JANE interpreter hence varies this weight according to distributions

\footnotetext{
${ }^{1}$ Proceedings of ASE 2001, San Diego, USA. Corresponding author. Department of Electrical \& Computer Engineering; 2356 Main Mall; Vancouver, B.C. Canada V6T1Z4. Phone: (604) 822-3381 Web: http: //tim.menzies.com.

${ }^{2}$ Dept. Computer Science \& Systems Analysis, Miami University, Oxford, Ohio, USA.
}

supplied by the analyst. After many such runs, a large log of possible behaviors is generated which is summarized by the TAR2 treatment learner.

Despite the range of possible actions within our encoding of CMM-2, and the subjectivity of the weights measures, there exists a small number of actions that have a significant impact on the project. Figure 1 shows three sets of actions learnt by TAR2. The left-hand-side histogram marked $\Delta_{0}$ shows the ratio of different project types predicted by our software management oracle. The other histograms $\left(\Delta_{i>0}\right)$ show how those ratios change after applying the treatments learnt by TAR2; i.e. managers taking certain actions to change their current situation. The worth of each option is a reflection of the proportion of good and bad projects, compared to $\Delta_{0}$; i.e. $\left(\right.$ worth $\left.\left(\Delta_{0}\right)=1\right)$. Note that as worth increases, the proportion of preferred projects also increases.

The rest of this paper describes how Figure 1 was generated.

\section{JANE}

A JANE programmer enters in models in a propositional rule-based language. Internally, these rules are converted into a directed graph with the following $\mathrm{BNF}^{1}$ :

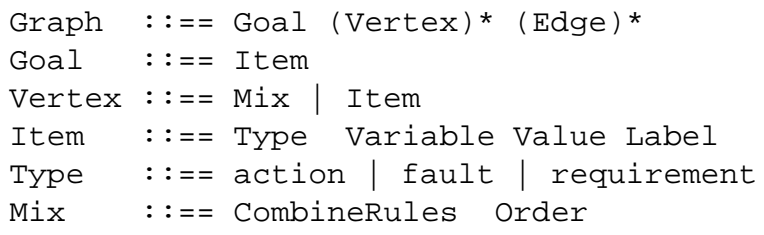

Note that each Graph has a special Goal Item. Conceptually, JANE is a backward chainer that performs a recursive descent from the Goal.

In JANE, every vertex is either an Item where some Value is assigned to some Variable; or a Mix vertex

\footnotetext{
${ }^{1}$ In this article's BNF notation, $\mathrm{W}::==\mathrm{X} Y \mid \mathrm{Z}$ denotes that the structure $W$ contains either the structures $\mathrm{X}$ and $\mathrm{Y}$ or the structure $Z$. (X) * denotes zero or more repeats of $X .(X)+$ denotes one or more repeats of $\mathrm{X}$. $\mathrm{X}$ ] denotes that $\mathrm{X}$ is optional. Terminals start with lower case, or are quoted
} 

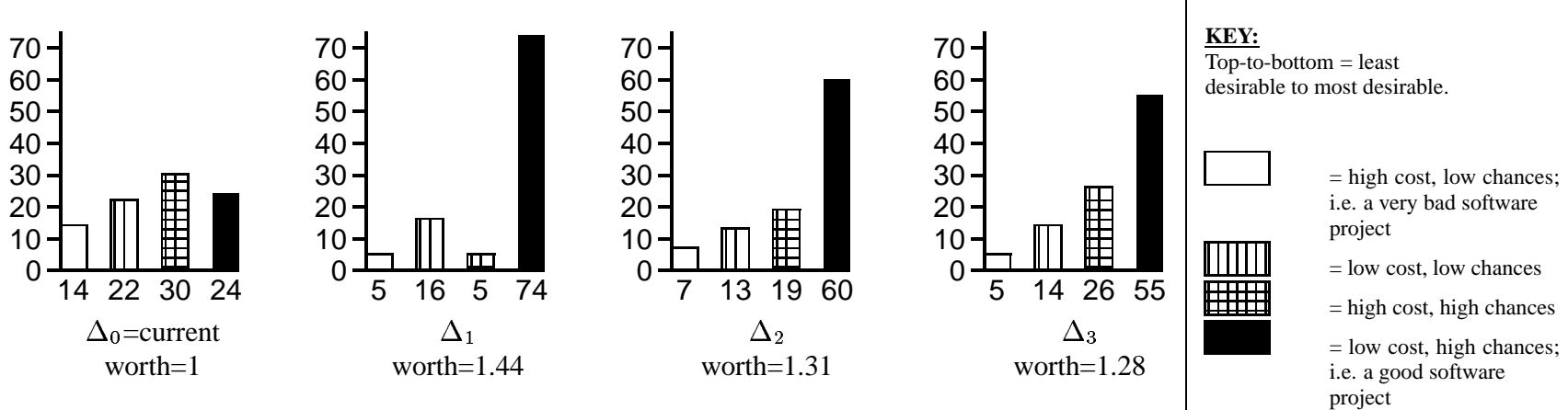

Figure 1. Ratios of different software project types seen in four situations.

where influences are combined. Each Vertex and Edge in JANE is augmented with a Cost and a Chances weight and Mix vertices define how Costs and Chances are combined"

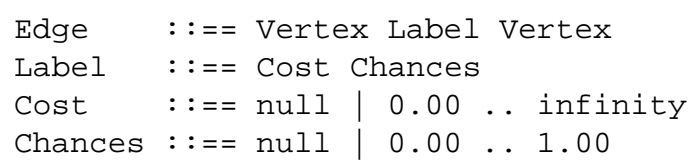

JANE's Chances define the extent to which a belief in one vertex can propagate to another. Costs let an analyst model the common situation where some of the Cost of some procedure is amortized by reusing its results many times. Hence, the first time we use an action we incur that Cost but afterwards, that action is free of charge.

The Cost and Chances of Vertexs are either provided by the JANE programmer or computed at runtime via a traversal of the edges. In JANE, this computation is defined by the CombineRules at the Mix vertices. For each child of a Mix node, a recursive descent is executed and the returned Cost and Chances values are combined according to the CombineRules:

$\begin{array}{ll}\text { CombineRules } \quad::= & \text { CostCombine } \\ \text { CostCombine } \quad::== & \text { first (cost) } \\ \text { ChancesCombine }::== & \text { first(chances) } \\ & \mid \begin{array}{l}\text { sum(chances) } \\ \text { negate } \\ \text { product (chances) }\end{array}\end{array}$

Negate is used for negation. For example, when searching $X$ if not $A$, the Chances of $X$ are 1-Chances $(A)$. Product (chances) is used for conjunctions. For example, when searching $X$ if $A$ and $B$ and $C$, the Chances of $X$ is the product of the chances of $A, B, C$. First (X) is used for simple disjunctive evidence. For example, when testing $X$ if $A$ or $B$ or $C$, then the Cost and Chances of $X$ is taken from the first member of $A, B, C$ that is satisficed. Sum $(\mathrm{X})$ is used for summing disjunctive evidence. For example, JANE supports a special operator called ors that is used for implementing disjunctive summing (JANE also supports several other novel operators, described in the next section). Ors is like or except that when testing (e.g.) $X$ if $A$ ors $B$ ors $C$, all members of $A, B, C$ will tested. If at least one succeeds, the the Cost and Chances of $X$ is summed from the satisficed members of $A, B, C$.

Sum (X) and Product (X) can be combined to implement risk mitigation effects. For example, suppose some action 5 disables most of the contribution of fault 3 on fault 1. In the JANE syntax, this can be coded as

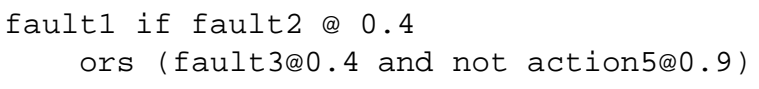

The ors operator uses Sum(chances); and uses Product (chances); and not uses negate. Hence, if the Chances of fault2, fault3, action 5 are all unity, then of action 5 is false, the Chances of fault 1 is $0.4+(0.4 *(1-$ $0))=0.8$. Alternatively, if action 5 is true, the Chances of fault 1 is $0.4+(0.4 *(1-0.9))=0.44 \ll 0.8$. That is, using action 5 significantly reduces the Chances of fault 1 .

\section{JANE and Random Search}

JANE supports two mechanisms for exploring the space of possibilities within the CMM-2 encoding. Both are random search tools. Firstly, when defining Costs and Chances, the programmer can supply a range and a skew. For example:

goodUnitTesting and cost $=1$ to +5

defines the cost of goodUnitTesting as being somewhere in the range 1 to 5 , with the mean skewed slightly towards 5 (denoted by the "+"). During a simulation, the first time this cost is accessed, it is assigned randomly according to the 
range and skew. The assignment is cached so that all subsequent accesses use the same randomly generated value. After each simulation, the cache is cleared. After thousands of simulations, JANE can sample the "what-if" behavior resulting from different assignments within the range.

Secondly, JANE can randomized where it searches using the Order of the Mix operators:

\begin{tabular}{ll|l|l|l|} 
Order & $::==$ Random & Left2Right \\
Left2Right & $::==$ and & or & ors & any $\mid$ not \\
Random & $::==$ rand | ror & rors & rany
\end{tabular}

In the case where the child-order represents some sequence that must be preserved (e.g. ordering software processes) JANE programmers can use Left 2 Right operators. However, repeatedly searching a graph in the same order may miss important interactions. In order not to miss such important interactions, JANE supports several random search operators: rand, ror, rors, and rany. For op $\in\{$ rand,ror $\}$, JANE tries to prove all of $X$ op $Y$ op $Z$, but does so in some randomly selected order.

Rany is best understood by comparison with ror. The expression $X$ ror $Y$ ror $Z$ is exited when any one of $X, Y, Z$ is satisficed. In contrast, JANE tries to prove one or more of $X$ rany $Y$ rany $Z$, and does so in some randomly selected order. Rany is useful when searching for subsets that contribute to some conclusion. For example, the following JANE rule offers several essential features of stableRequirements plus several optional factors relating to monitoring change in evolving projects. The essential features are rand-ed together while the optional factors are rany-ed together.

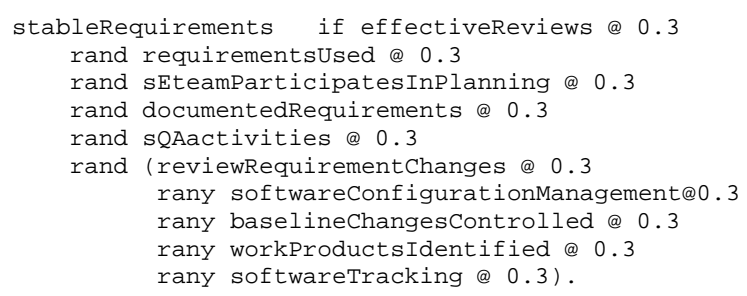

Rors is useful for specifying the high-level goals of the system. While rany will search some subset of its parameters, rors will search all its parameters. For example, in this less-than-perfect world, it is unlikely we can be rich and happy and healthy, but that should not stop us from trying; i.e.

goal if rich rors happy rors healthy

The operators rand, ror, rors and rany have the same satisficing criteria as and, or, ors and any respectively, but the latter search left-to-right while the former search in a randomly selected order. In terms of the CombineRules described above, And and rand are conjunctive operators; Or and ror are simple disjunctive operators; and the rest are summing disjunctive operators.

\section{The CMM2 Model}

CMM2 written in JANE has 55 Items with Value $\epsilon$ $\{t, f\}$. Of the 55 Items, 27 were identified as management actions that could be changed by managers (see Figure ??). The top-level Goal was goodProject.

Within the model, Chances values were added to 79 edges of the 150 edges in the model. While each of these values are based on expert judgement, their precise value is subjective. Hence, each such Chances value $X$ was altered to be a range

chances $=0.7 * x$ to $1.3 * X$

so the simulator could experiment with values nearby the stated Chances value.

The full model is available via email from the authors.

\section{Learning From JANE}

One execution of JANE can be summarized by the Cost and Chances of the top-level goal goodProject, the 79 values assigned to the subjective edges, and the values assigned to the 27 manager's actions. Random walking over JANE models can hence generate an overwhelming amount of data $(79+27+2=108$ data points per simulation, times the number of simulations).

Summarization of JANE output is performed by the TAR2 treatment learner. A treatment learner outputs a set of possible treatments $(\Delta)$ using the process described below. Each treatment is assessed by the change it makes to the distribution of classes. A good treatment increases the frequency of the better classes (where "better" is defined below).

Treatment learners input a set of classified examples $(E g)$. Each example is a conjunction of attribute ranges, plus one classification $c$. This classification comes from a pre-defined set of classes $C$; i.e. $c \in C$. Each classification $c$ is associated with a numeric score i.e. some classifications score better than others.

For the CMM model, after 2000 random walks, a wide range of generated Costs and Changes in goodProject were observed. These ranges were sub-divided into high/low bands of roughly the same size. Combing high/low Cost/Chances yields four classes. These classes were scored as follows:

Score=0: High cost, low chance

Score=1: Low cost, low chance

Score=2: High cost, high chance

Score $=4$ : Low cost, high chance.

That is, our preferred projects are cheap and highly likely while expensive, low odds projects are to be avoided. 
A treatment learner searches for candidate attribute ranges; i.e. ranges that are more common in the best classification than in the not-so-best classification. In the CMM domain, such a candidate is an attribute range that would tend to drive the system into low cost, high chance projects.

A heuristic ranking $\delta$ is given to each candidate reflecting (i) how much more common is the candidate in the best class than in some non-best-class $c$; and (ii) how much better is the best class than class $c$ :

$\delta=(\operatorname{score}($ best $)-\operatorname{class}(c)) *\left(\frac{\mid E g \cap A=R \cap \text { class }=\text { best } \mid}{\mid E g \cap A=R \cap \text { class }=c \mid}\right)$

where $A=R$ denotes an attribute $A$ with range $R$ and $|E g \cap X|$ denotes the size of the subset of examples that contain $X$.

Candidates with a large $\delta$ score are attribute ranges that are far more frequent in the best class (high chances, low cost projects) than in other classes. The treatments $\Delta$ are all subsets of candidates with a ranking higher that some user-specified $\delta$ threshold value $\delta_{T}$.

In reality, it is hard to change many aspects of a project and the wise software engineer seeks the smallest number of changes with the greatest impact. Assuming we seek three changes, there are 560 possible treatments of size 3 in our 16 candidates. The best treatment is the one that offers the biggest improvement to the class distributions seen in the untreated data. To find the best treatment, we compute following ratio for all 560 treatments:

$$
\operatorname{worth}\left(\Delta_{i}\right)=\frac{\sum_{c \in C} \mid E g \cap \Delta_{i} \cap \text { class }=c \mid * \operatorname{score}(c)}{\text { baseline worth }}
$$

where baseline worth is the worth seen in the untreated example set:

$$
\text { baseline worth }=\sum_{c \in C} \mid E g \cap \text { class }=c \mid * \operatorname{score}(c)
$$

\section{Results}

Figure 2 shows the three best treatments $\left(\Delta_{1}, \Delta_{2}, \Delta_{3}\right)$ found using this technique (and Figure 1 compared the effects of these treatments to the untreated examples). Note that the values of each attribute are reported using the tags no, lower, middle, or upper. In treatment learning, continuous attribute ranges are divided into $\mathrm{N}$-discrete bands based on percentile positions. For $\mathrm{N}=3$, we can name the bands lower, middle, upper for the lower, middle, and upper $33 \%$ percentile bands.

In Figure $2, \Delta_{2}$ and $\Delta_{3}$ are advising to lower the cost of:

Using requirements: This could be accomplished by (e.g.) by sharing them around the development team in some searchable hypertext format
$\Delta_{1}:$ requirementsUsed.Cost $=$ lower and not periodicSoftwareReviews and formalReviewsAtMilestones. Cost =lower

$\Delta_{2}$ : requirementsUsed.Cost $=$ lower and goodUnitTesting. Cost $=$ middle and formalReviewsAtMilestones. . Cost $=$ lower

$\Delta_{3}:$ goodUnitTesting. Cost $=$ lower and periodicSoftwareReviews. Cost $=$ middle and formalReviewsAtMilestones. Cost $=$ lower

\section{Figure 2. The three best treatments found in the CMM-2 model.}

Performing formal reviews at milestones: This could be accomplished by (e.g.) using ultra-lightweight formal methods such as proposed by Leveson [2].

Performing good unit testing: This could be accomplished by (e.g.) hiring better test engineers.

The value no refers to missing values; a report of $X=n o$ is a recommendation not to use $X$ as part of a treatment. Such a negative recommendation is seen in $\Delta_{1}$ which is advising against periodicSoftwareReviews (plus lowering the costs of using requirements and formal reviews at milestones). Note that if periodicSoftwareReviews are conducted, $\Delta_{3}$ is saying that there is no apparent need to reduce the cost of such reviews.

\section{DISCUSSION}

Space limitations prevent a review of work related to this research such as the goal-graphs of Chung et.al. [1] (such a review is available from the authors). This discussion section will hence focus on the major threat to the external validity of the above conclusions.

The knowledge source used in this study (CMM2) is widely, but not universally, accepted. Proponents of some other knowledge source could reject the specifics of the above conclusions, but still use the general technique; i.e. they could encode their preferred knowledge source in JANE and repeat the above analysis.

\section{References}

[1] L. Chung, B. Nixon, E. Yu, and J. Mylopoulos. NonFunctional Requirements in Software Engineering. Kluwer Academic Publishers, 2000.

[2] N. Leveson, S. Cha, and T. Shimall. Safety verification of ada programs using software fault trees. IEEE Software, 8(7):4859, July 1991.

[3] M. Paulk, C. Weber, B. Curtis, and M. Chriss. The Capability Maturity Model: Guidelines for Improving the Software Process. Addison-Wesley, 1995. 